This item was submitted to Loughborough's Research Repository by the author.

Items in Figshare are protected by copyright, with all rights reserved, unless otherwise indicated.

\title{
Towards a decision-support framework for reducing ramp-up effort in plug- and-produce systems
}

\author{
PLEASE CITE THE PUBLISHED VERSION
}

PUBLISHER

IEEE

\section{VERSION}

AM (Accepted Manuscript)

\section{PUBLISHER STATEMENT}

(C) 2019 IEEE. Personal use of this material is permitted. Permission from IEEE must be obtained for all other uses, in any current or future media, including reprinting/republishing this material for advertising or promotional purposes, creating new collective works, for resale or redistribution to servers or lists, or reuse of any copyrighted component of this work in other works.

\section{LICENCE}

All Rights Reserved

\section{REPOSITORY RECORD}

Zimmer, Melanie, Pedro Ferreira, Paul Danny, Ali Al-Yacoub, Niels Lohse, and Valerio Gentile. 2019. "Towards a Decision-support Framework for Reducing Ramp-up Effort in Plug-and-produce Systems". figshare. https://hdl.handle.net/2134/37415. 


\section{Towards a Decision-support Framework for Reducing Ramp-up Effort in Plug-and-Produce Systems}

\author{
Melanie Zimmer, Pedro Ferreira, Paul Danny, \\ Ali Al-Yacoub, Niels Lohse \\ Wolfson School of Mechanical, Electrical and Manufacturing \\ Engineering, Loughborough University, \\ Loughborough, UK - LE11 3TU \\ \{M.Zimmer2, P.Ferreira, P.Danny, A.Al-Yacoub, \\ N.Lohse\}@lboro.ac.uk
}

\author{
Valerio Gentile \\ We Plus S.p.A. \\ Strada Torino 43/95 \\ 10043 Orbassano (TO), Italy \\ valerio.gentile@we-plus.eu
}

\begin{abstract}
Nowadays, shorter and more flexible production cycles are vital to meet the increasing customised product demand. As any delays and downtimes in the production towards time-to-market means a substantial financial loss, manufacturers take an interest in getting the production system to full utilisation as quickly as possible. The concept of plug-andproduce manufacturing systems facilitates an easy integration process through embedded intelligence in the devices. However, a human still needs to validate the functionality of the system and more importantly must ensure that the required quality and performance is delivered. This is done during the ramp-up phase, where the system is assembled and tested first-time. System adaptations and a lack of standard procedures make the ramp-up process still largely dependent on the operator's experience level. A major problem that currently occurs during ramp-up, is a loss of knowledge and information due to a lack of means to capture the human's experience. Acquiring this information can be used to simplify future ramp-up cases as additional insights about change actions and their effect on the system could be revealed. Hence, this paper proposes a decisionsupport framework for plug-and-produce assembly systems that will help to reduce the ramp-up effort and ultimately shorten ramp-up time. As an illustrative example, a glueing station developed as part of the European project openMOS is considered.
\end{abstract}

Keywords - decision-support framework; ramp-up; plug-andproduce; expert system; learning.

\section{INTRODUCTION}

In the context of a globally competitive market, it is vital for companies to rapidly respond to their customers' needs by transferring products quicker from the design phase into full production. Adapting existing or introducing new products, usually requires some form of modification to the production system. Many efforts to shorten this period in industry have, however, proven to be insufficient [1]. These physical and process adaptations occur during the ramp-up period of the product lifecycle and can take in the automotive sector, for example, up to several months. While a variety of definitions for the term ramp-up exists, for the purpose of this paper, ramp-up is the activity that delivers systems which operate to an established quality and performance level. As finetuning of equipment and process adjustments need to take place during that phase, the need for human intervention is required [2]. But this and the absence of knowledge about the system and its operational behaviour, result in poorly understood and lengthy ramp-up processes [19]. Therefore, as most of the complexity is dealt with by the operator, the effectiveness of the ramp-up is largely dependent on the operator's level of expertise and

The reported work is part of the openMOS project partially funded by the European Commission as part of the EC-H2020-IA (GA 680735). knowledge. In spite of this, the visibility of decisions taken during that phase is often not given as the user's decisions are currently not captured. This means that a lot of knowledge that could facilitate future ramp-ups is lost.

Plug-and-produce systems are expected to bring advances in the way data are made available. For one, automation devices can be rapidly introduced into production through a level of embedded intelligence. Moreover, a cyber-physical representation of the manufacturing system enables a high degree of agility. The idea of plug-and-produce manufacturing systems is not new. Two examples are European research projects such as IDEAS [3], where the adaptability of assembly equipment was proven, and PERFoRM [4], which targeted the need for increased flexibility and reconfigurability in the manufacturing domain. Applying the concept of plug-and-produce manufacturing systems to the ramp-up phase has yet not been addressed.

The European project 'open Dynamic Manufacturing Operating System for Smart 'Plug-and-Produce' automation components' (openMOS) [5] targets the development of an innovative, openly accessible Plug-and-Produce (P\&P) system platform. As part of openMOS, this work aims to contribute to the growing area of research in plug-and-produce assembly systems by proposing a decision-support framework that can increase the sustainability of these systems during ramp-up. This is envisioned to be achieved by bringing operational data and human knowledge together. Relevant information will be made available in a way that the operator's decision-making for quick system adaptations is supported. It is expected that this will lead to reduced ramp-up time while simultaneously increasing the level of achieved performance during production.

The remaining part of the paper proceeds as follows: a brief literature review is presented in Section II to back the problem definition in Section III. The decision-support framework is explained in Section IV, followed by an illustrative example in Section V. Finally, the conclusion in Section VI provides a summary and an outlook for future work.

\section{RELATED WORK}

Shortening ramp-up time has previously been considered in literature. Du et al. [1] present a methodology that allows the identification of system faults during ramp-up through the comparison of predicted and actual operational data. In [6], Terwiesch et al. describe a case study for production ramp-up and changeover in the hard disk drive industry, where a production process to be brought to full-scale production is assessed under consideration of quality and cost. 
Even though ramp-up is a crucial step for manufacturing systems, difficulties already arise in the definition of the term ramp-up and its boundaries. For instance, rather than achieving high-volume production, the end of the ramp-up phase can be defined based on certain objective achievements, such as product quality [7], [8]. In [9], Doltsinis, et al. propose a framework for measuring production performance during ramp-ups. Their framework introduces a one-dimensional ramp-up performance index that combines the performance metrics equipment functionality, output quality and performance optimisation (including productivity, quality and time). While durations, processes, and tools for ramp-up might differ, common characteristics have been widely discussed. Surbier et al. [10] summarise their findings in their state-of-the-art review as follows: (1) little information and preliminary knowledge about the product and the process is available, (2) tools and methods used for pilots are insufficient, and training for personnel might not be possible, (3) a lack of communication and cooperation, as well as trust issues among personnel exist, (4) production output and capacities, are low, but demand and cycle times are usually higher, (5) disturbances to product quality, process and supply chain occur frequently and uncertainty is high.

The uncertainties that exist during ramp-up make the use of mathematical models difficult. In data-driven modelling, a model can be created with only limited information about the physical behaviour of the system based on collected measurements [11]. Uncovering unknown links between the input and output values of a system is the major role of learning in data-driven modelling. Machine learning has been widely applied in manufacturing to help uncover hidden patterns in data [12] with one area of application in the field of decision-support. Many machine learning approaches, however, rely on the availability of sufficient data samples to provide meaningful insights into reoccurring patterns. Although the development of machine learning techniques in ramp-up is currently still immature, Reinforcement Learning (RL) and Recommendation Systems (RS) have been applied to ramp-up scenarios. Doltsinis and Lohse [13] examine the practicality of more formal machine learning methods to assist decision-making during ramp-up. They investigate the efficiency of a Monte Carlo approach that applies reinforcement learning to a ramp-up scenario emulating Terwiesch and Xu's [14] copy-exactly strategy of an assembly station. Another approach based on reinforcement learning was studied by Doltsinis et al. [2] where time savings can be achieved using a Q-learning algorithm. Willmann et al. [15] present the use of knowledge-based production ramp-up process (K-RAMP) for ramping-up a new product considering the production objectives and product specifications. Based on process knowledge, product requirements and operator's expertise, the authors propose the use of an RS that provides rapid access to necessary product and process knowledge. These systems can also automatically extract relevant knowledge and generalise well for new processes. For creating recommendations as part of their decision-support framework, Scrimieri et al. [16] use a variant of the k-nearest neighbour algorithm in their work. The framework is targeted to the ramp-up process of modular assembly systems and learns configuration as well as tuning factors by considering past experience.

Doltsinis et al. [9] highlight that the non-repetitiveness of the ramp-up phase increases the complexity of the process. This is because decisions about the logical or physical adjustments of the system are often made on a try and error basis. Nevertheless, Ball et al. [17] recognise the opportunity of being able to obtain performance improvements during ramp-up. This is seen in the introduction and first-time testing of novel production processes. In their paper, Fjällström et al. [8] study the source and type of information that can aid to enable production ramp-up. The authors find that in order to deal with critical events during ramp-up, information about the domain and problem at hand are particularly useful. It is also reported that differences between novice and experienced staff exist. Namely, less knowledgeable personnel can draw from less domain knowledge and might have more difficulties in its application. The authors conclude that personal knowledge is of great value in ramp-up scenarios and can save effort and time. Li and Rajagopalan [18] highlight how knowledge impacts production quality and cost, and emphasise that system setup times are influenced by learning. Terwiesch and Bohn [19] conduct experiments (induced learning) that, on the one hand, reduce production capacity in a short period of time, while on the other, model process improvements in form of learning. This is done to analyse the interactions between learning, capacity utilisation, and yields. The authors use dynamic programming techniques to derive value and cost of experiments. They find that long-term investment in learning often suffers from short-term cost improvements and that keeping the level of experimentation constant during ramp-up is not ideal.

Glock and Grosse [20] review the literature on the topic of decision-support models for ramp-up. The authors state that process improvements leading to increased productivity and deduced errors are the results of learning that takes place during the ramp-up activity. Improving the aspect of knowledge capture and communication during ramp-up is an important research subject, helping to keep disturbances caused by the loss of human knowledge as small as possible. Yet few researchers propose a suitable learning approach and assisting tools (software) for ramp-up [21].

\section{PRoblem Definition}

Despite all the good work, insufficient capture and reuse of human knowledge still contribute to the increased effort necessary for ramping-up a system. For this research work, it is hypothesised that additional insights can be gained from the human's knowledge. Therefore, a means for gathering and communicating this data needs to be in place.

While plug-and-produce manufacturing systems reduce the ramp-up effort through their level of modularity and intelligence, they do not deal with all the complexities emerging in system configurations. Nevertheless, the added functionalities and availability of information in P\&P systems do offer new opportunities to address the ramp-up process. In fact, this links to well-established concepts from multiple advances in P\&P, which provide so-called Skills, Skill Requirements and Recipes [22], [23]. Simply put, these concepts are used to describe an assembly process with an underlying relationship of the Product, Process and Resource (PPR). A Skill represents assembly process capability essential for the execution of a process by the equipment. Depending on the level of granularity, skills can either be atomic or composite. An atomic skill describes elementary skills such as pick and place, while a composite skill comprises these lower level skills to more complex ones. Skills fulfil a set of Skill Requirements needed for the manufacture of a product. The execution of skills requires the 


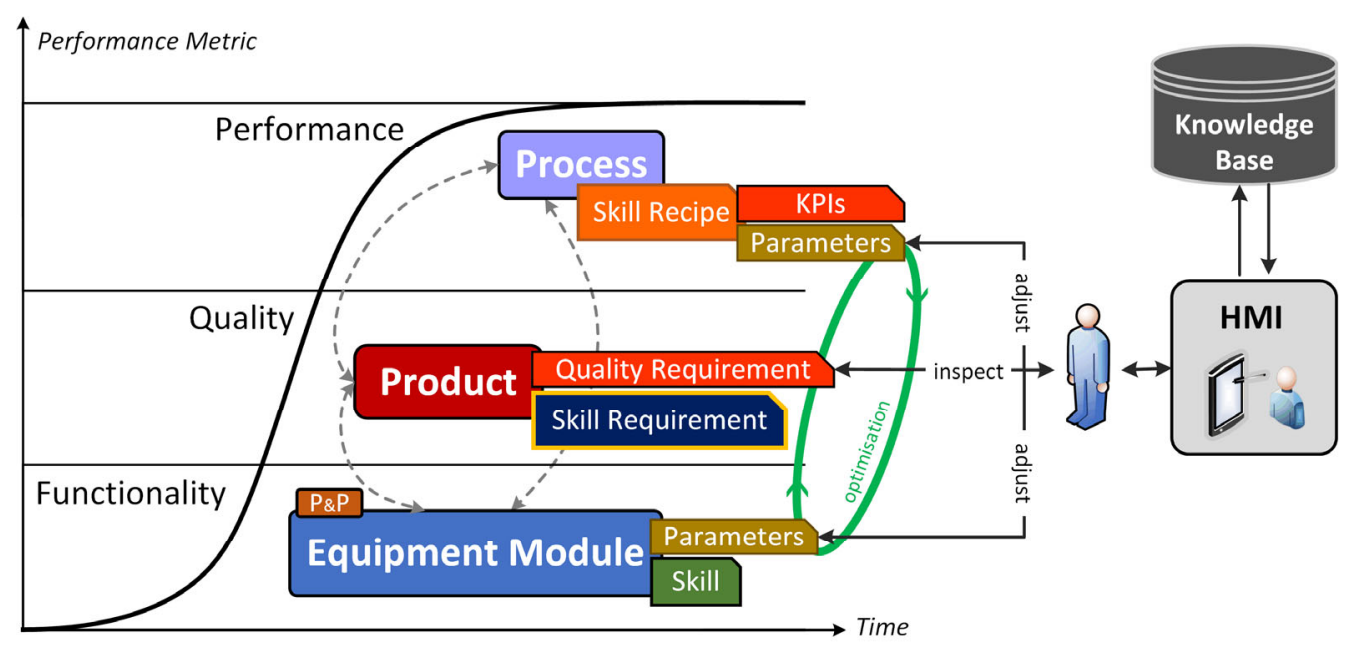

Fig. 1. Overview of Problem Definition.

instantiation of its execution parameters defined as a Recipe. The Recipe can be linked to the fulfilment of a set of skill requirements, which enable the creation of products. These models are used in the openMOS project to provide easy access to data and structured information about the assembly process, product, and equipment.

Referring to the performance metrics proposed in [9], ramp-up can be divided into three parts: functionality, quality, performance. Linking this to a P\&P context (Fig. 1), the first step is to guarantee that the system functionality is provided. This means that all the equipment modules need to be set up accordingly and the skills need to be tested. The next part of ramp-up foresees the introduction of target products into the system. For this, the operator also must guarantee that for each product the right recipes in the form of software and hardware settings to deliver the product exist. After the functioning step of the ramp-up is guaranteed, the product quality needs to be assured given the product description. In cases where the quality does not meet the required level, equipment and process adjustments done by the human will be essential. Once the product quality is ensured, the system performance under consideration of production targets is considered. As potentially several recipes can be defined, fine-tuning these input parameters within a valid range can bring performance improvements. In order for the human to be able to decide whether the performance targets are reached, easy access to system information and Key Performance Indicators (KPIs) are required. Any changes made at that point can also have an effect on the product quality again, so a reassessment will be needed. Optimal quality and performance targets might not be able to be reached simultaneously and a trade-off between these two metrics is most likely.

In this context, this work proposes a decision-support framework for ramp-up. It is designed to leverage data from this phase to assist the operator in making decisions that will lead to shortened ramp-up effort and, ultimately, reduced time. As the ramp-up is still very human-centric, the operator must be fully integrated into the framework. Reviewing the literature implied that the application of machine learning models to the ramp-up case can create a knowledge base that can guide the human in the absence of experience through future reconfigurations and product introductions. Such a framework must be able to work within the particular nature of the ramp-up process. For example, the uniqueness of systems and low availability of data during ramp-up have to be taken into account for choosing suitable data-driven decision models.

\section{DECISION-SUPPORT FRAMEWORK}

The intended decision-support framework (Fig. 2) aims to better integrate the operator into the ramp-up activity. For this, the framework contains means to facilitate the data collection throughout the different stages of ramp-up. Through the use of learning mechanisms, knowledge extraction can be achieved based on the obtained data. In guiding the operator through these actions, the framework will allow to more rapidly bring the system to an operational state.

For this work, ramp-up is understood as a sequence of different system states which capture the system settings at a given time. At the end of this sequence, a working system that performs to the established requirements is delivered. Between the individual system states, different actions can repeatedly be chosen and executed by the human until the required functionality, quality and performance are achieved. These actions can be classified into the following stages: assessment stage, change stage and test stage. During these stages, various operation, sensor and human-related data will be collected and stored in the openMOS Cloud. Data gathering on its own is not be very meaningful, particularly since production systems tend to be quite different. Therefore, it is important to gather the data in a structured and contextualised manner. The openMOS project uses in particular models for the product, equipment, execution, and assembly process that cater for the collection of data for plug-and-produce assembly systems. However, these models do not include the necessary elements for depicting human actions and their results on the system. As the operator's knowledge and expertise can reveal insights about the ramp-up process that cannot be easily identified through the operational data or setup alone, it will be key to acquire information on human actions and resulting system behaviour to enable the capture human expertise. For that reason, additional semantic models have been defined to encapsulate equipment and process adjustments as well as human knowledge in the form of assessments and observations.

Assessing the current state is necessary to decide whether the required performance targets are achievable or have been already met under the given setting. An assessment is a rating 


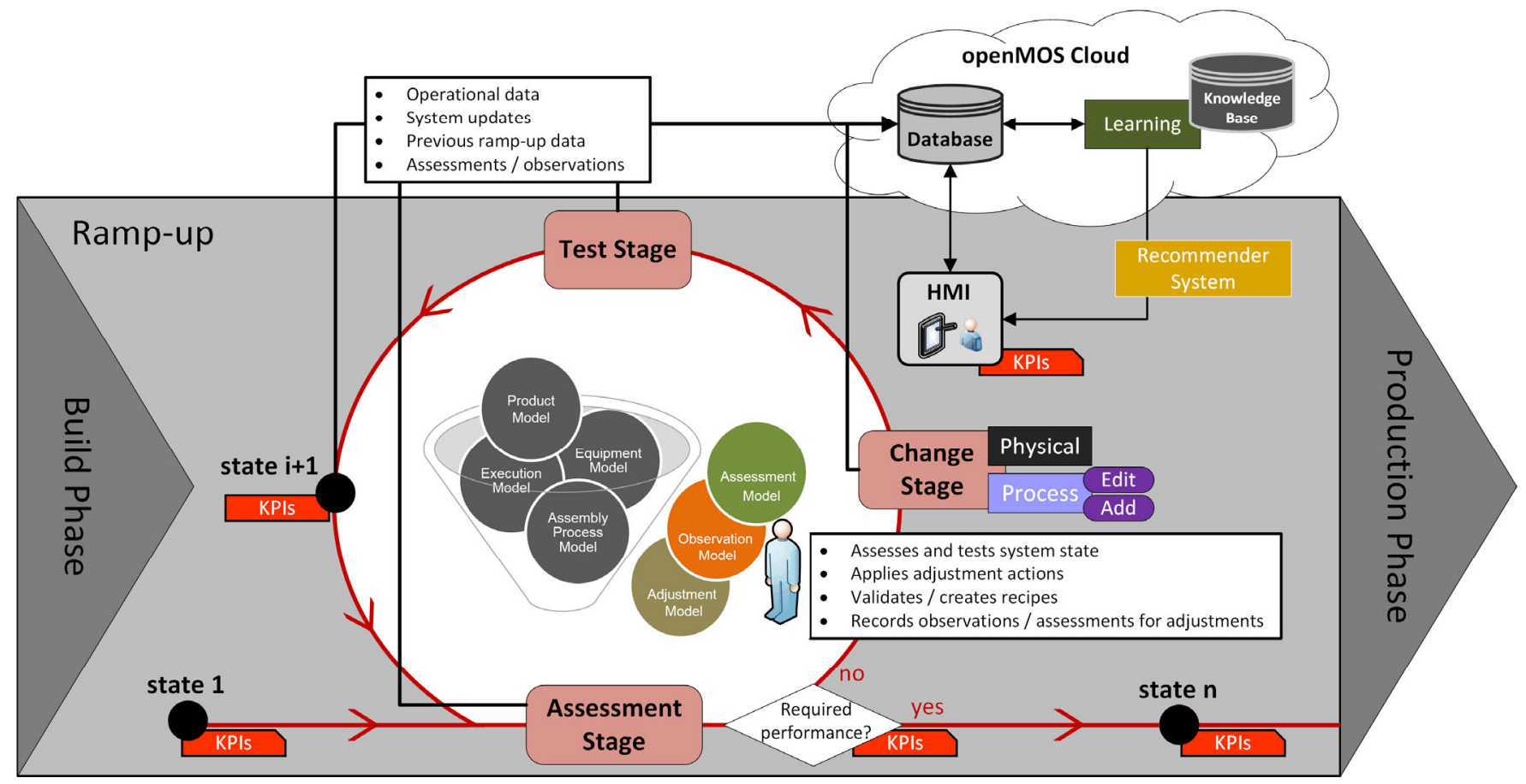

Fig. 2. Overview of proposed decision-support framework for ramp-up.

of functionality, quality, and performance that will be provided by the operator. In the case where the assessment shows that the system does not yet deliver the expected functionality, product quality and performance, either an equipment or process adjustment has to be made. All the interaction between the system and the operator is facilitated through a Human- Machine Interface (HMI) that has been developed as part of the openMOS project. So is, for example, an overview of the system, its individual equipment modules, and status provided including details about available skills and recipes, order instances and product lists offered. It is noted, that the operator can add observations about the functionality, quality or performance of an equipment to the openMOS system at any time of the ramp-up cycle. An optional comment in the form of free-form text can be added to the observation. This allows the provision of additional visibility about the system.

Although this visibility can already speed up the ramp-up phase, the envisioned framework aims to further enhance this process by proposing potential improvement actions. Once a change is indicated, the Recommender System (RS) will be triggered to check for any recommendations related to the equipment or process under consideration. Recommendations are made accessible to the user through the HMI and can be described as a list of different suggested change actions and an overall evaluation based on predicted functionality, quality, and performance. For this work, it is intended to realise Recommender Systems to identify similar recipes in the historical knowledge data and then propose adjustment actions based on previous user assessments. In order to provide such recommendations, a hybrid RS will be developed to aid the decision-making during the ramp-up processes. This hybrid RS system will be composed of content-based and collaborative filtering models. The contentbased model aims to determine the most similar recipe in the historical data, while the collaborative model aims to identify the most appropriate adjustment action. The Recommender System will map the input recipes to a set of actions that can be executed by the operator to enhance the overall assessment score for the system performance. The mapping is achieved through a training procedure, which includes data collection, features extraction, model parameters optimisation and testing. At the beginning of the ramp-up process, it is expected that the recommendations will be less accurate due to the lack of sufficient data. However, as the ramp-up progresses, more and more data become available. All the information gathered by the system as well as information provided by the human is stored in the openMOS Cloud. The obtained data then serve as an input to data-driven models. This allows building a sufficient knowledge base over time so that recommendations will gradually improve.

Once the operator has decided to undertake a change action and applied the alterations to the system, the system will be tested under this new configuration, resulting in a new system state. During the test stage, the operator will run a test execution for a skill, recipe or product on the current system setup. After this stage has been completed, a new system state including relevant KPIs exists and will again be required to be assessed by the operator. The different stages, i.e. assessment, change, and test, are repeated as long as the required production targets have not been deemed reached and accepted by the operator.

\section{ILLUSTRATIVE EXAMPLE}

On the basis of a physical demonstrator from the openMOS project, the concepts of the previously described decision-support framework are illustratively explained. The general operational task of this demonstrator is an industrial glueing process as part of an assembly step demonstrator.

The glueing station consists of standard industrial components that have been retrofitted with plug-and-produce capabilities. The main component of the physical setup is a single ABB IRB120 6-axis industrial robot, which is connected to an IRC5 controller. A two-finger gripper has been fixed to the robot to hold a flat metal workpiece. An automated time-pressure dispensing unit (Fisnar JB1113N) is used. Glue is dispensed in a controlled manner through a 


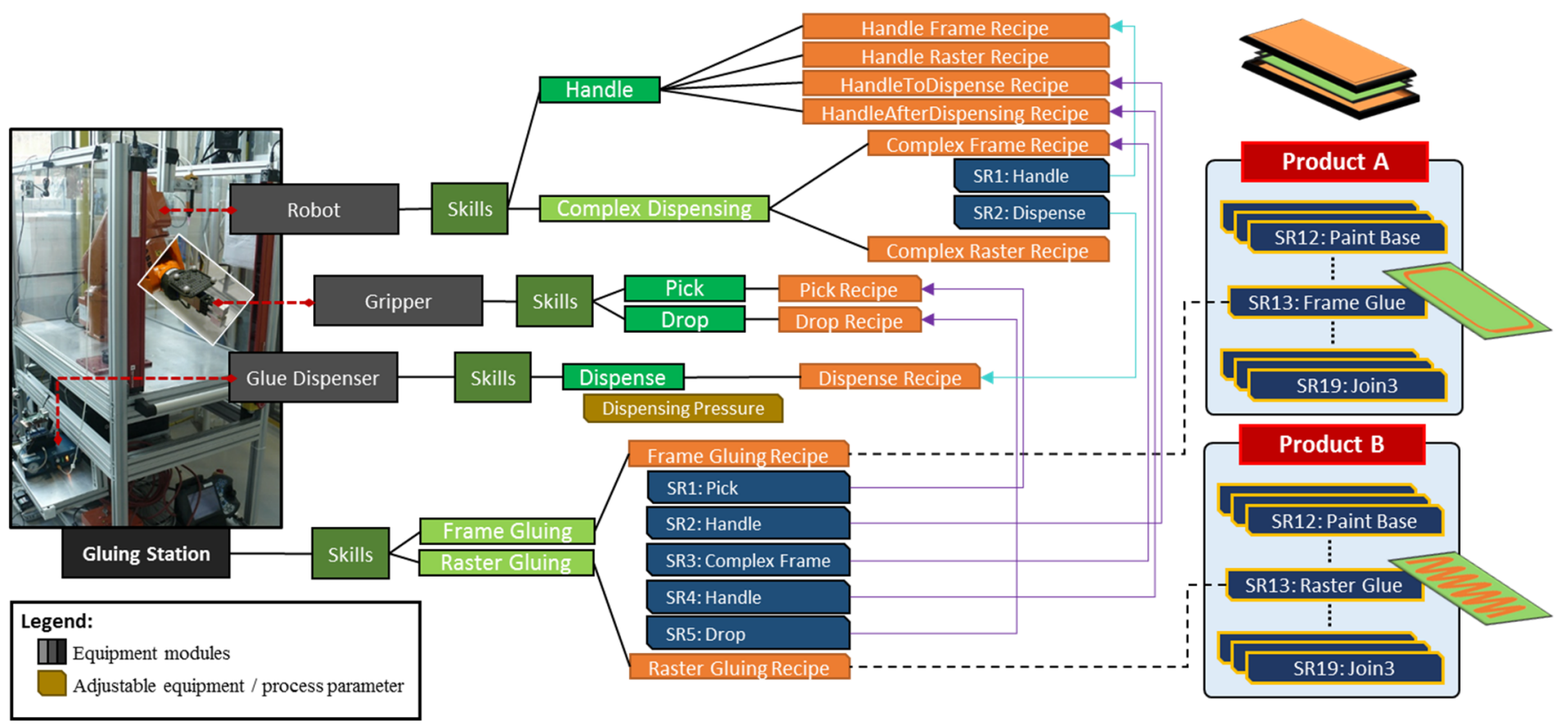

Fig. 3. Simplified overview of the cyber-physical representation of the glueing station and products under consideration.

nozzle that has been mounted to the surrounding frame as the robot will manipulate the metal workpiece. It is to mention, that the demonstrator will be used in the future to collect initial ramp-up data to evaluate the effectiveness of the proposed framework. Therefore, additional sensors providing environmental and energy consumption information have been fitted to the glueing workstation.

To prepare for the capture of process parameterisation and equipment adjustments, the glueing station has been mapped to its cyber-physical module representations as pictured in Fig. 3. The atomic skills for this workstation consist of pick, release, handle and dispense. At the higher hierarchical level, the composite skills of the workstation are raster glueing skill and frame pattern skill. For better readability, the skill requirements, which establish the composite skill for raster glueing, have been omitted but can be assumed to be similar to the skill requirements for the frame glueing composite skill. For both, processes and equipment modules, specific parameters can be selected to allow for a certain range of adjustment. In the given case, for example, the handle skill for the robot can be parameterised for operating the robot at different speeds, i.e. 20, 30, and $40 \mathrm{~mm} / \mathrm{s}$. For the glue dispenser, the dispense skill can take on the values 10,16 , or 20 psi for setting the dispensing pressure. Moreover, the dispensing nozzle can be physically replaced to gain different line widths of $1.2 \mathrm{~mm}$ or $1.6 \mathrm{~mm}$. If any of the process or equipment parameters are, however, not set fittingly, the desired product quality and performance will not be achieved. This is why ramping-up a system is such an important activity, as here all equipment and process parameters will be tweaked until this is the case.

For this use case, two product variations (Product A and B) of an electronic package housed in a $3 \mathrm{D}$ printed enclosure are considered. The products can generally differ in the electronic packaging paint, glueing pattern, and enclosure. As the focus is on the glueing process, it is assumed that Product $\mathrm{A}$ and $\mathrm{B}$ at least vary in the glueing pattern that is applied to the product base. The two possible glueing options that are available are a frame and raster pattern. For simplicity, the focus for this demonstrator is solely on the process parameterisation and equipment adjustments. This means that for each pattern one or more recipes defining the glueing path can exist and a fitting interaction between the nozzle diameter, dispensing pressure and robot speed needs to be found.

As an exemplary walk-through of how the proposed decision-support system is foreseen to help an operator during ramp-up, three outcomes of the glueing process can be seen in Fig. 4. The first result is achieved through a parameterisation of 10 psi. After a visual inspection by the operator, the produced pattern is deemed undesirable. This means that the raster glueing recipe has not been instantiated with the correct value for the dispensing pressure. When changing this value now through the HMI, the Recommender System will provide the operator with certain change actions, as shown in Fig. 5. As the decision about a suggested change lies with the operator, recommendations provided by the system cannot just be accepted but also discarded. This information, in addition to whether an advised change was actioned, will be taken into consideration for future learning steps to improve the quality of the recommendations. For the purpose of this example, it is assumed that the operator chooses to modify the dispensing pressure to 20 psi next. Once the change has been recorded, the raster glueing recipe is executed. The second run does also not provide the anticipated result as the line, in this case, turns out to be too thick for the intended purpose. Again, a change is initiated by the operator and the Recommender System will have updated its suggestions based on the latest data collected. In the last instance, the dispensing pressure is set to $16 \mathrm{psi}$, which results in a satisfactory outcome. It is noted that for all the steps undertaken, the operator can choose to add an observation, describing, for example, the produced outcome or reasons for actions taken in more detail.

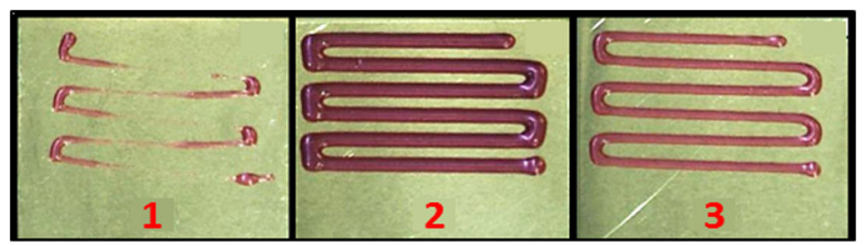

Fig. 4. Exemplary outcomes of the glueing process [24]. While outcome 3 represents the desired outcome, 1 and 2 show unacceptable quality results (discontinuity of line; dispensed line too thick). 


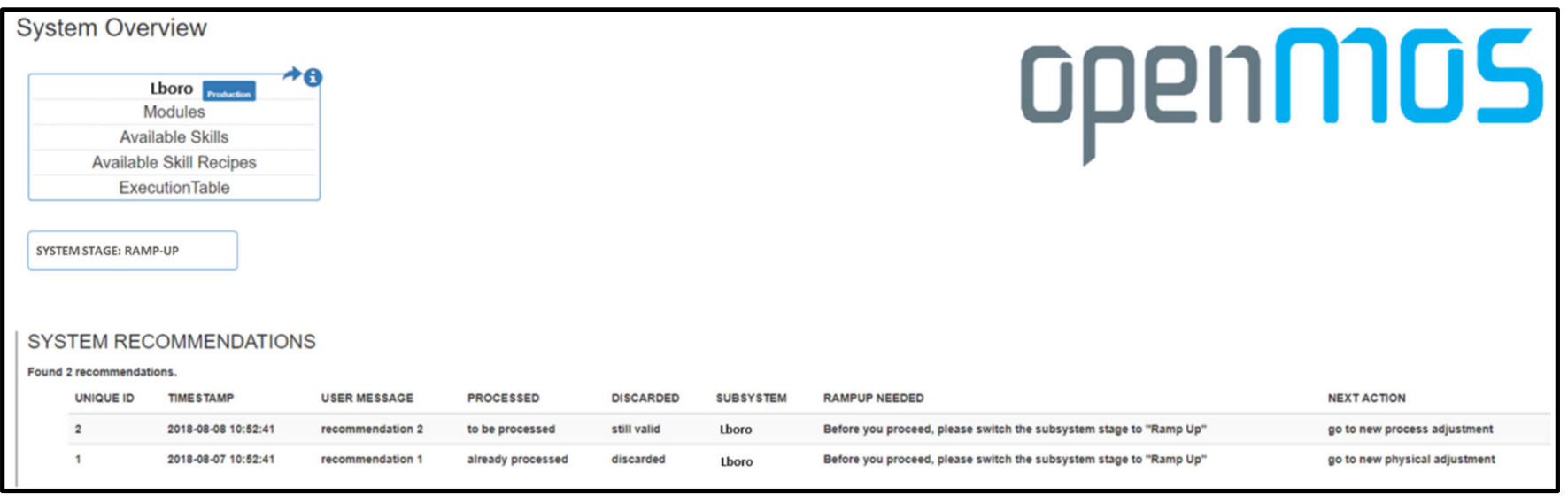

Fig. 5. HMI view for recommended actions.

\section{CONCLUSION}

In this paper, a framework to aid an operator in the decision-making during the ramp-up of plug-and-produce assembly systems is proposed. The framework sets out to reduce ramp-up time and effort through learning from rampup actions and experience. For this, data-driven models will be leveraged to provide assistance in the form of recommended change actions to the human. An example of how the framework is intended to work is described with the help of an illustrative case for an assembly glueing step. As a first step of practically creating this framework, existing models for plug-and-produce assembly systems from the European project openMOS have been enhanced to capture the ramp-up data. In particular, models for representing equipment and process adjustments as well as human observations and assessments have been developed. Future work foresees to implement a hybrid Recommender System consisting of content-based and collaborative filtering models for which the characteristics of ramp-up, such as low data volume, have to be studied. The framework will be initially tested and evaluated by ramping up the glueing workstation described in this paper. However, to ensure the robustness and flexibility of the framework, other industrial use cases and ramp-up scenarios will be considered in the future.

\section{ACKNOWLEDGEMENT}

The reported work is part of the openMOS project partially funded by the European Commission as part of EC-H2020-IA (GA 680735). The support is gratefully acknowledged. Additional thanks go to the EPSRC Centre for Doctoral Training in Embedded Intelligence (grant number EP/L014998/1) for the funding of closely related work.

\section{REFERENCES}

[1] S. Du, L. Xi, J. Ni, P. Ershun, and C. R. Liu, "Product lifecycle-oriented quality and productivity improvement based on stream of variation methodology," Comput. Ind., vol. 59, no. 2-3, pp. 180-192, 2008.

[2] S. Doltsinis, P. Ferreira, and N. Lohse, "Reinforcement learning for production ramp-up: A Q-batch learning approach," Proc. - 2012 11th Int. Conf. Mach. Learn. Appl. ICMLA 2012, vol. 1, pp. 610-615, 2012.

[3] "IDEAS." [Online]. Available: www.ideas-project.eu/.

[4] "PERFoRM." [Online]. Available: www.horizon2020-perform.eu/.

[5] “openMOS." [Online]. Available: www.openmos.eu/about/vision/.

[6] C. Terwiesch, R. E. Bohn, and K. S. Chea, "International product transfer and production ramp-up: A case study from the data storage industry," $R$ D Manag., vol. 31, no. 4, pp. 435-451, 2001.

[7] J. Fleischer, D. Spath, and G. Lanza, "Quality simulation for fast ramp up," in Proceedings of the 36th CIRP International Seminar on Manufacturing Systems, 2003.
[8] S. Fjällström, K. Säfsten, U. Harlin, and J. Stahre, "Information enabling production ramp-up," J. Manuf. Technol. Manag., vol. 20, no. 2, pp. 178-196, 2009.

[9] S. C. Doltsinis, S. Ratchev, and N. Lohse, "A framework for performance measurement during production ramp-up of assembly stations," Eur. J. Oper. Res., vol. 229, no. 1, pp. 85-94, 2013.

[10] L. Surbier, G. Alpan, and E. Blanco, "A comparative study on production ramp-up: State-of-the-art and new challenges," Prod. Plan. Control, vol. 25, no. 15, pp. 1264-1286, 2014.

[11] P. Kadlec, B. Gabrys, and S. Strandt, "Data-driven Soft Sensors in the process industry," Comput. Chem. Eng., vol. 33, no. 4, pp. 795-814, 2009.

[12] D. T. Pham and A. A. Afify, "Machine-learning techniques and their applications in manufacturing."

[13] S. C. Doltsinis and N. Lohse, "A model-free reinforcement learning approach using Monte Carlo method for production Ramp-up policy improvement - A copy exactly test case," in IFAC Proceedings Volumes (IFAC-PapersOnline), 2012, vol. 14, no. PART 1, pp. 16281634.

[14] C. Terwiesch and Y. Xu, "The copy-exactly ramp-up strategy: Tradingoff learning with process change," IEEE Trans. Eng. Manag., vol. 51, no. 1, pp. 70-84, 2004.

[15] R. Willmann, S. Biffl, and E. Serral Asensio, "Determining Qualified Production Processes for New Product Ramp-up using Semantic Web Technologies," in Proceedings of the 14th International Conference on Knowledge Technologies and Data-Driven Business (I-KNOW), 2014.

[16] D. Scrimieri, R. F. Oates, and S. M. Ratchev, "Learning and reuse of engineering ramp-up strategies for modular assembly systems," $J$. Intell. Manuf., vol. 26, no. 6, pp. 1063-1076, 2015.

[17] P. D. Ball, S. Roberts, A. Natalicchio, and C. Scorzafave, "Modelling production ramp-up of engineering products," Proc. Inst. Mech. Eng. Part B J. Eng. Manuf., vol. 225, no. 6, pp. 959-971, 2011.

[18] G. Li and S. Rajagopalan, "Process Improvement, Quality, and Learning Effects," Manage. Sci., vol. 44, pp. 1517-1532, 1998.

[19] C. Terwiesch and R. E. Bohn, "Learning and process improvement during production ramp-up," Int. J. Prod. Econ., vol. 70, pp. 1-19, 2001.

[20] C. H. Glock and E. H. Grosse, "Decision support models for production ramp-up: A systematic literature review," Int. J. Prod. Res., vol. 53, no. 21, pp. 6637-6651, 2015.

[21] K. Konrad, M. Hoffmeister, M. Zapp, A. Verl, and J. Busse, "Enabling fast ramp-up of assembly lines through context-mapping of implicit operator knowledge and machine-derived data," in IFIP Advances in Information and Communication Technology, 2012, vol. 371 AICT, pp. 163-174.

[22] D. Semere, J. Barata, and M. Onori, "Evolvable Assembly Systems: Developments and Advances," in IEEE International Symposium on Assembly and Manufacturing, 2007, pp. 282-287.

[23] K. Agyapong-Kodua, C. Haraszkó, and I. Németh, "Recipe-based Integrated Semantic Product, Process, Resource (PPR) Digital Modelling Methodology," Procedia CIRP, vol. 17, pp. 112-117, 2014.

[24] K. Elgeneidy, A. Al-Yacoub, Z. Usman, N. Lohse, M. Jackson, and I. Wright, "Towards an automated masking process: A model-based approach," Proc. Inst. Mech. Eng. Part B J. Eng. Manuf., 2018. 